ACCEPTED MANUSCRIPT • OPEN ACCESS

\title{
An extremely simplified optics laboratory for teaching the fundamentals of Fourier analysis
}

To cite this article before publication: Marco Potenza 2020 Eur. J. Phys. in press https://doi.org/10.1088/1361-6404/abd4c9

\section{Manuscript version: Accepted Manuscript}

Accepted Manuscript is "the version of the article accepted for publication including all changes made as a result of the peer review process, and which may also include the addition to the article by IOP Publishing of a header, an article ID, a cover sheet and/or an 'Accepted Manuscript' watermark, but excluding any other editing, typesetting or other changes made by IOP Publishing and/or its licensors"

This Accepted Manuscript is $\odot 2020$ The Author(s). Published by IOP Publishing Ltd..

As the Version of Record of this article is going to be / has been published on a gold open access basis under a CC BY 3.0 licence, this Accepted Manuscript is available for reuse under a CC BY 3.0 licence immediately.

Everyone is permitted to use all or part of the original content in this article, provided that they adhere to all the terms of the licence https://creativecommons.org/licences/by/3.0

Although reasonable endeavours have been taken to obtain all necessary permissions from third parties to include their copyrighted content within this article, their full citation and copyright line may not be present in this Accepted Manuscript version. Before using any content from this article, please refer to the Version of Record on IOPscience once published for full citation and copyright details, as permissions may be required. All third party content is fully copyright protected and is not published on a gold open access basis under a CC BY licence, unless that is specifically stated in the figure caption in the Version of Record.

View the article online for updates and enhancements. 


\title{
An extremely simplified optics laboratory for teaching the fundamentals of Fourier analysis
}

\author{
M A C Potenza \\ Department of Physics, University of Milan, Milan, Italy \\ E-mail: marco.potenza@unimi.it \\ Received xxxxxx \\ Accepted for publication $\mathrm{xxxxxx}$ \\ Published xxxxxx
}

\begin{abstract}
Easy and cheap optics experiences are described to teach undergraduate students the fundamental properties of Fourier transform on an experimental basis. By exploiting the eye as the Fourier transforming device, a common magnifying lens and quasicoherent light from a small white LED, quantitative results can be easily obtained about the fundamental theorems in Fourier analysis. The concept of coherence is also introduced in an elementary way. This approach has been successfully adopted to teach third year students in Physics, who operated in a complete autonomous way during the COVID-19 lockdown without accessing any laboratory. This proves the effectiveness of the method. Thanks to its experimental simplicity it can be easily extended to teach and show the fundamental effects to a larger audience, including high school students.
\end{abstract}

Keywords: Fourier Optics, Diffraction, Optics Laboratory

\section{Introduction}

Fourier analysis fundamentals are of utmost importance for undergraduate students. Examples are ubiquitous and it is common to address physical and mathematical concepts in many fields, as evidenced for example in [1]. Learning from practical applications, as it is possible in laboratory, represents a further advantage complementing the most traditional, formal approaches. More than ten years teaching Fourier analysis in a dedicated optics laboratory gave strong evidence of this advantage. The COVID-19 lockdown in 2020 immediately demanded for a complete revisitation of the experimental approaches, usually based on a well-equipped optics laboratory. This implied an extreme simplification of the experiences for students, eventually enabling them to operate independently with an almost inessential, very cheap instrumentation. The present work stems from the experience with students of the third-year laboratory course for the Master degree in Physics. Despite the important limitations of this experimental approach, it represents an example of how simple an experiment can be made, putting everybody in conditions to perform quantitative observation with results similar to what is usually done in the laboratory. Experiences are so simple to be suitable for secondary school students, too, even if a complete quantitative approach might be better understood from undergraduate students.

In the following, basic elements of Fourier analysis and a few fundamentals of Optics are firstly introduced, fixing the formalism adopted across the entire work. The formalism is kept close to the 
book of Goodman [2], which teachers and students can refer to as a guide for the arguments treated within this work, as well as for further insights about a number of issues, exercises and interesting applications in many fields. A brief introduction is given about the concept of spatial coherence and the way to generate light in a proper way to approach the experiences. The naked eye is then introduced as the basic Fourier transforming "device" to measure Fraunhofer diffraction patterns of some case examples. The basic theorems of Fourier analysis are then reproduced and quantitatively discussed through proper experiences performed with common objects and materials. A colored photographic filter can be used for better quantitative analysis. After that, with the aid of a simple magnifying lens, the integral Fourier theorem is reproduced, with the naturally consequent applications to spatial filtering of wavefronts, imaging of phase objects and the discussion of the Abbe-Porter experiments. By introducing specific objects, easily obtained with a traditional photographic film, additional experiences can be performed with a better quantitative analysis. Perspectives of further experiences and applications are discussed.

\section{Fourier analysis fundamentals}

Linearity is a property shared by a number of physical phenomena. Basically, the advantage of linear systems is the ability to obtain the behavior of a system to a given stimulus as the linear combination of the behaviors to "elementary" stimuli. Fourier analysis is a fundamental tool to deal with linear systems, and Optics represents an ideal stage to represent its basic properties: hence the name Fourier Optics. Two main classes of systems can be distinguished, either or not the light is endowed with a property called spatial coherence (see below). In the former case light is described by wavefronts represented as complex-valued functions; in the latter real-valued intensity distributions are used. Here we focus at introducing the fundamentals of Fourier analysis of linear systems with complex-valued functions. The real-valued case can be obtained as a special case, as it can be found in many textbooks ([2] for example). Optical systems, specifically diffractive optics, naturally needs to adopt two-dimensional (2D) Fourier analysis. This comes from the general description of wavefronts, that are complex-valued functions of two independent variables, i.e. defined on a 2D domain.

Provided that the necessary existence conditions are guaranteed, a given function $g(x, y)$ can be decomposed in its Fourier components, "elementary" functions of the form $\exp \left[i 2 \pi\left(f_{\mathrm{X}} x+f_{\mathrm{Y}} y\right)\right]$. Here $i$ is the imaginary unit and $f_{\mathrm{X}}$ and $f_{\mathrm{Y}}$ are the spatial frequencies along the coordinate directions $x, y$. Such functions are simple plane waves, with the peculiar property of maintaining the spatial frequencies constant across the $(x, y)$ plane. Across this plane, the lines of constant phase have equation:

$$
y=-\frac{f_{\mathrm{X}}}{f_{\mathrm{Y}}} x+\frac{m}{f_{\mathrm{Y}}}
$$

with $m$ integer. Spacing is given by $d=1 / f_{0}$, where $f_{0}^{2}=f_{\mathrm{X}}^{2}+f_{\mathrm{Y}}^{2}, f_{0}$ being the spatial frequency of the plane wave, with $1 / f_{0}>\lambda$. The elementary function introduced above can be interpreted as the section in the $(x, y)$ plane of a three-dimensional plane wave. The plane wave has wavefronts perpendicular to a direction forming angles $\alpha, \beta, \gamma$ with the three coordinate axes. These angles are defined by the three direction $\operatorname{cosines} A=\cos \alpha=\lambda f_{\mathrm{X}}, B=\cos \beta=\lambda f_{\mathrm{Y}}, C=\cos \gamma=\lambda f_{\mathrm{Z}}$. Notice that $C$ is fixed by the relation $C=\sqrt{1-A^{2}-B^{2}}$. The complex amplitude of the plane wave in the whole space $(x, y, z)$ is completely fixed by the elementary function in plane $(x, y)$. Finally, it can be easily shown that the plane wave is propagating through space forming an angle $\vartheta$ with the optical axis z according to the relation $\sin \vartheta=\lambda f_{0}(\vartheta$ is complementary to the angle formed with the $(x, y)$ plane, which is that defining the direction cosines). For small diffraction angles this expression can be approximated as $\vartheta \sim \lambda f_{0}$, that will be useful below.

We attract the reader's attention to the following basic, complementary observations.

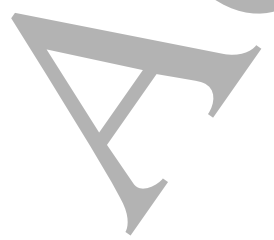


1) A (thin) grating imposing a sinusoidal amplitude modulation with spatial frequency $f_{0}$ will generate two simmetrically diffracted plane waves only (see [2]) propagating at angles $\pm \vartheta$, fulfilling the elementary grating relation:

$$
d \sin \vartheta=m \lambda \quad m= \pm 1
$$

Therefore, we conclude that $\sin \vartheta=\lambda f_{0}$, the same expression obtaind above for the plane wave. More precisely, if $f_{0}$ has components $f_{\mathrm{X}}$ and $f_{\mathrm{Y}}$, then the diffracted plane waves will have spatial frequencies $\pm f_{\mathrm{X}}$ and $\pm f_{\mathrm{Y}}$. The peculiarity of this grating is represented by the existence of one diffracted order, at variance with common gratings that produce several or many plane waves upon diffraction. This is strictly related to the elementary function introduced above as the basic Fourier component: the sinusoidal grating represents the essence of diffraction, being simultaneously the simplest function for decomposing any amplitude modulation and the modulation generating one diffracted order. Any amplitude modulation $A(x, y)$ can be viewed as the superposition of sinusoidal gratings: the amplitude and phase values, $A\left(f_{\mathrm{X}}, f_{\mathrm{Y}}\right)$, give the Fourier transform of $A(x, y)$, and the superposition of the corresponding diffraced fields gives the diffraction pattern of the whole modulation itself.

2) If the plane wave of above would superimpose to a unit-amplitude plane wave (reference wave) propagating along the optical axis $z, \exp [i k z]$, and they interfere onto a screen perpendicular to $z$, the interference term will give intensity modulations described by:

$$
\delta I(x, y)=2 \mathfrak{N}\left[\exp \left[i 2 \pi\left(f_{\mathrm{X}} x+f_{\mathrm{Y}} y\right)\right]\right]=2 \cos \left[2 \pi\left(f_{\mathrm{X}} x+f_{\mathrm{Y}} y\right)\right]
$$

The interference pattern will be the "footprint" of the plane wave on the screen. This is also called the hologram of the plane wave: if illuminated by a unit-amplitude plane wave identical to the reference one, it will generate a copy of the incoming plane wave $\exp \left[i 2 \pi\left(f_{\mathrm{X}} x+f_{\mathrm{Y}} y\right)\right]$, propagating at an angle $\vartheta$ such that $\sin \vartheta=\lambda f_{0}$, plus a "new" plane wave diffracted at symmetric angle, $-\vartheta, \exp \left[i 2 \pi\left(-f_{\mathrm{X}} x-f_{\mathrm{Y}} y\right)\right]$. Holography just means "complete drawing" in Greek and is named after its ability to "draw" amplitude and phase of the incoming waves, although uncertainty remains about the sign of the phase.

This is what is needed to figure out the basics of Fourier analysis through elementary optics experiences. In the following we will focus on the ability of simple lenses to produce Fourier transforms, and exploit this to reproduce the fundamental theorems. In order to introduce the formalism and language, we first recall the basic properties of lenses. For simplicity we will refer here to the book of Goodman [2], of which we follow the formal approach that is particularly clear in introducing Fourier analysis.

\section{Fourier transforming properties of lenses}

A thin lens is considered here as the simplest optical element imposing a spherical shape to a plane wave that passes through. Such a lens in the plane $(\xi, \eta)$ will impose a phase modulation:

$$
U(\xi, \eta)=\exp \left[-i \frac{k}{2 f}\left(\xi^{2}+\eta^{2}\right)\right]
$$

where $f$ is the focal length. Let be an "object" imposing an amplitude and phase modulation described by the complex transmission function $T(x, y)$. Just beyond the object, the wavefront will be described by the same function. By using the Huygens-Fresnel integrals, and neglecting phase terms, in the focal plane $(x, y)$ of the lens we obtain:

$$
U(x, y)=\frac{1}{\lambda f} \iint T(\xi, \eta) \exp \left[-i \frac{2 \pi}{\lambda f}(x \xi+y \eta)\right] \mathrm{d} \xi \mathrm{d} \eta
$$


Positions $(x, y)$ in the focal plane identify angles $\vartheta_{\mathrm{X}}=x / f, \vartheta_{\mathrm{Y}}=y / f$, that can be converted into spatial frequencies as introduced above: $f_{\mathrm{X}}=x / \lambda f, f_{\mathrm{X}}=x / \lambda f$. By substituting into the previous expression:

$$
U\left(f_{X}, f_{Y}\right)=\frac{1}{\lambda f} \iint T(\xi, \eta) \exp \left[-i 2 \pi\left(\xi f_{\mathrm{X}}+\eta f_{\mathrm{Y}}\right)\right] \mathrm{d} \xi \mathrm{d} \eta
$$

Apart for phases, it is the Fourier transform of the function $T(x, y)$. The intensity distribution at the focal plane will just provide the power spectral density of $T(x, y)$, that is the squared modulus of its Fourier transform. We stress that this result shows the plane wave decomposition of the transmission function, but it has been rigorously obtained on the basis of the Huygens-Fresnel approach, that stems from the description of wavefronts through spherical elementary waves.

We can also cast things as a very simple consequence of the basic geometrical optics. In fact, the fundamental lens equation reltaes the positios of the conjugate planes at distances $p$ and $q$ from a lens of focal length $f$ (see Fig. 1 ): $\frac{1}{p}+\frac{1}{q}=\frac{1}{f}$ : at the focal plane, i.e. for $q=f$, the corresponding conjugate is set at infinity. This means that for the image at the focal plane, the object is solely described by angular positions. Therefore, a given position $(x, y)$ in the focal plane will be illuminated by the intensity of a plane wave entering the lens with angles $\vartheta_{X}$ and $\vartheta_{Y}$ defined above, that correspond to spatial frequencies $\left(f_{\mathrm{X}}, f_{\mathrm{Y}}\right)$ : this gives the power spectrum of the wavefront.

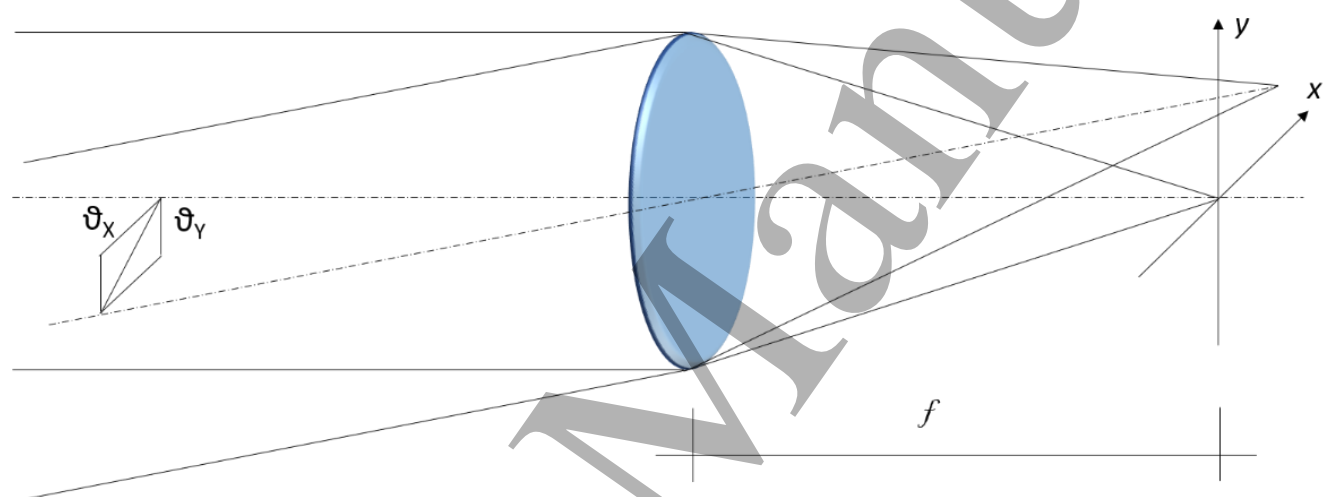

Figure 1. Imaging an object at infinity: the image is focused on the focal plane of the lens. Positions on this plane correspond to angular positions (directions) of the plane waves entering the lens.

A further insight into the Fourier spectrum can be given with a simple lens. A wavefront described by the function $T(\xi, \eta)$ of above is collected by a lens and light propagates downstream to a plane $(u, v)$. Both planes are at a distance $f$ from the lens, that is placed at the plane $(x, y), z=0$. By exploiting again the Huygens-Fresnel intergrals and plane wave propagation, it can be easily shown that the field complex amplitude in the plane $(u, v)$ is the exact Fourier transform of the field complex amplitude in plane $T(\xi, \eta)$. In symbols:

$$
U(u, v)=\mathfrak{F}[T(\xi, \eta)]
$$

As above, coordinates $(u, v)$ plays the role of spatial frequencies: $f_{\mathrm{X}}=u / \lambda f, f_{\mathrm{Y}}=v / \lambda f$. The formal proof of this fundamental result can be found for example in [2]. We will return to this peculiar property of lenses below, when discussing the integral Fourier theorem. 
We stress that in all the imaging systems operating with the object at infinity, the intensity profile of the image is nothing else that the power spectral density of the wavefronts entering the objective. We base our experiences upon this very simple result.

\section{Spatial coherence}

Using a lens as a Fourier transformer needs to transilluminate the object with a "perfect" plane wave, as it comes from the theoretical description of above. Light emitted by common sources, including cheap laser pointers, does not meet these criteria. Nevertheless, a simple, non-trivial argument exhibits the solution in the concept of spatial coherence, which is briefly recalled here below to introduce the experimental requirements for performing simple experiments with common white light sources. Basic elements about coherence can be found for example in $[1,3,4]$, while more detailed approaches are given in [5] and [6]. A remarkable application of the concept of spatial coherence arguments has been given with the Michelson stellar interferometer [7,8].

Coherence can be interpreted - or just defined - as the ability of a wave to form (stable) interference fringes. The stability of the interference fringes can be only obtained with stationary relative phase. Two distinct concepts can be made: spatial coherence and temporal coherence, depending whether the correlations are considered among two points in space (fixed time) or in time (same position). The advent of laser, and more recently of extrememly cheap laser sources, made temporally coherent light easily available for many elementary optics experiences. Nevertheless, most of them have been originally perfomed with natural light, the actual need being spatial, instead of temporal, coherence.

Let's consider a radiation source extended over a distance $D$. Radiation passes through a couple of pinholes a distance $d$ one from the other and a distance $\mathrm{z}$ far from the source (see Fig. 2.a). At a distance $z^{\prime}$ from the pinholes a screen is placed to observe the superposition of the emerging fields. Let $z^{\prime}$ be large enough to allow the radiation diffracted from the pinholes to superimpose. Each point $\mathrm{P}$ of the source generates a field that, upon diffraction by both pinholes, forms Young's interference fringes at the screen. The position of the interference maxima and minima depend on the relative phases of the diffracted waves, i.e. ultimately on the relative positions of the emitter within the source and the two pinholes. In the case of an ideal pointlike source the Young's fringes are always visible. Otherwise, whether interference fringes will sum up or cancel out is a nontrivial question to be addressed. From a formal point of view, we define the mutual intensity of the field at positions $\boldsymbol{r}_{1}, \boldsymbol{r}_{2}$ as $[1,5]$ :

$$
\Gamma\left(\boldsymbol{r}_{1}, \boldsymbol{r}_{2} ; \Delta t\right)=\left\langle U\left(\boldsymbol{r}_{1}, t\right) U^{*}\left(\boldsymbol{r}_{1}, t\right)\right\rangle
$$

Where \langle\rangle indicates time averaging over a time much longer than the wave period. By introducing the local intensities $I_{1}=\left|U\left(\boldsymbol{r}_{1}\right)\right|^{2}$ and $I_{2}=\left|U\left(\boldsymbol{r}_{2}\right)\right|^{2}$, the quantity $\gamma=\frac{\Gamma}{\sqrt{I_{1} I_{2}}}$ provides the fringe visibility, or contrast [6].

The minimum distance between the pinholes such that the relative phase of the emerging waves changes by $2 \pi$ rad is said transverse coherence length. This is also the condition for forming stable interference fringes onto the screen at $z^{\prime}$, so that we can redefine the coherence length as the minimum distance between the pinholes such that the visibility of the interference fringes vanishes. In other terms, the coherence length can just be interpreted as the minimum spacing of the interference fringes formed on the screen by the superposition of any couple of pointlike emitters at the source. Provided that the pinhole distance is smaller than the spacing of the interference fringes formed by wavefronts $W_{1}$ and $W_{2}$ from the two furthest emitters in positions $x_{1}$ and $x_{2}$ (Fig. 2.b), the two pinholes are illuminated by a field with fixed phase. This phase can also change with time, even very fast depending on the emission processes, but the relative phase of the fields at the two apertures is always fixed, and such is the position of the interference fringes at the 
observation screen. The spacing between the interference fringes formed at the pinholes by waves from $x_{1}$ and $x_{2}$ is simply given by:

$$
\Lambda=\frac{\lambda}{\sin \theta} \sim \frac{\lambda}{\theta} \sim \frac{\lambda}{D} Z
$$

where $\theta$ is the angle subtended by points , $x_{1}$ and $x_{2}$, that is also the angular aperture of the source observed from the pinhole position. Eq. (9) comes from a very fundamental theorem, obtained by Van-Cittert and Zernike independently and named after both. It provides the field-field, two-points correlation function of the field at a distance $z$ from a source of linear extension $D$ as the Fourier transform of the source transverse intensity profile.

a)

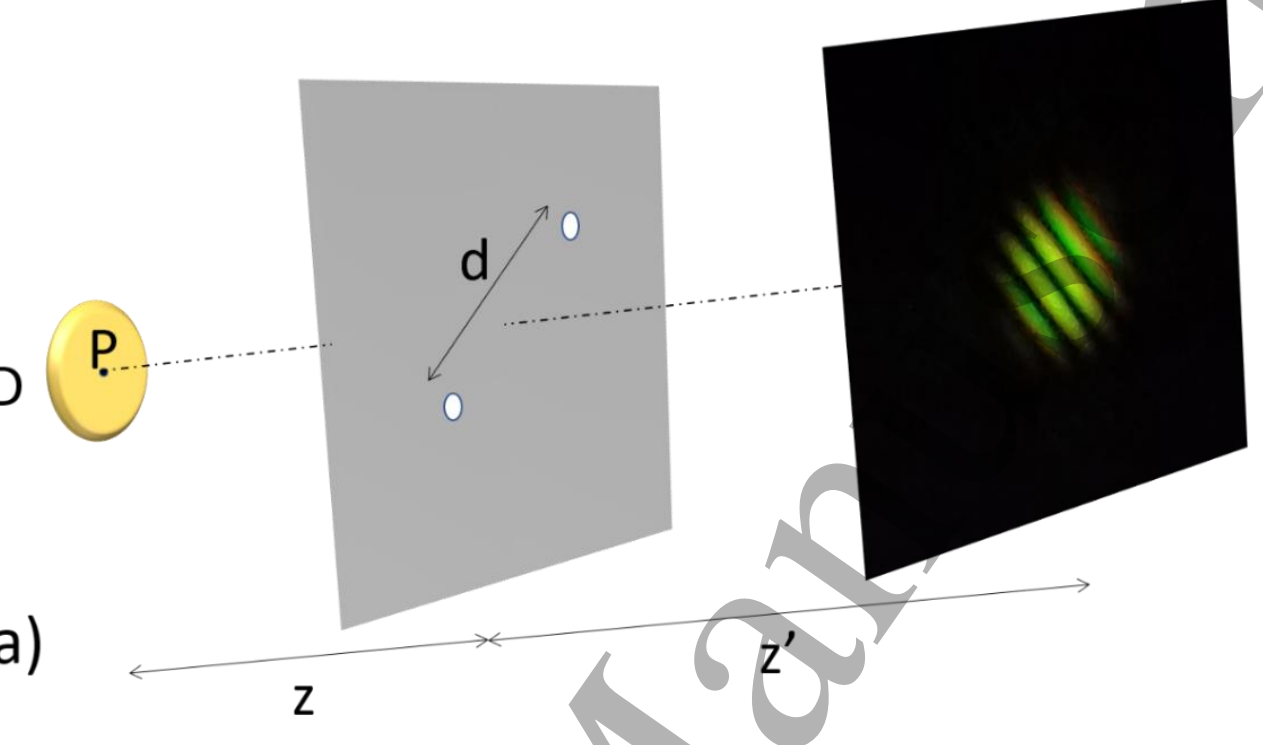

b)

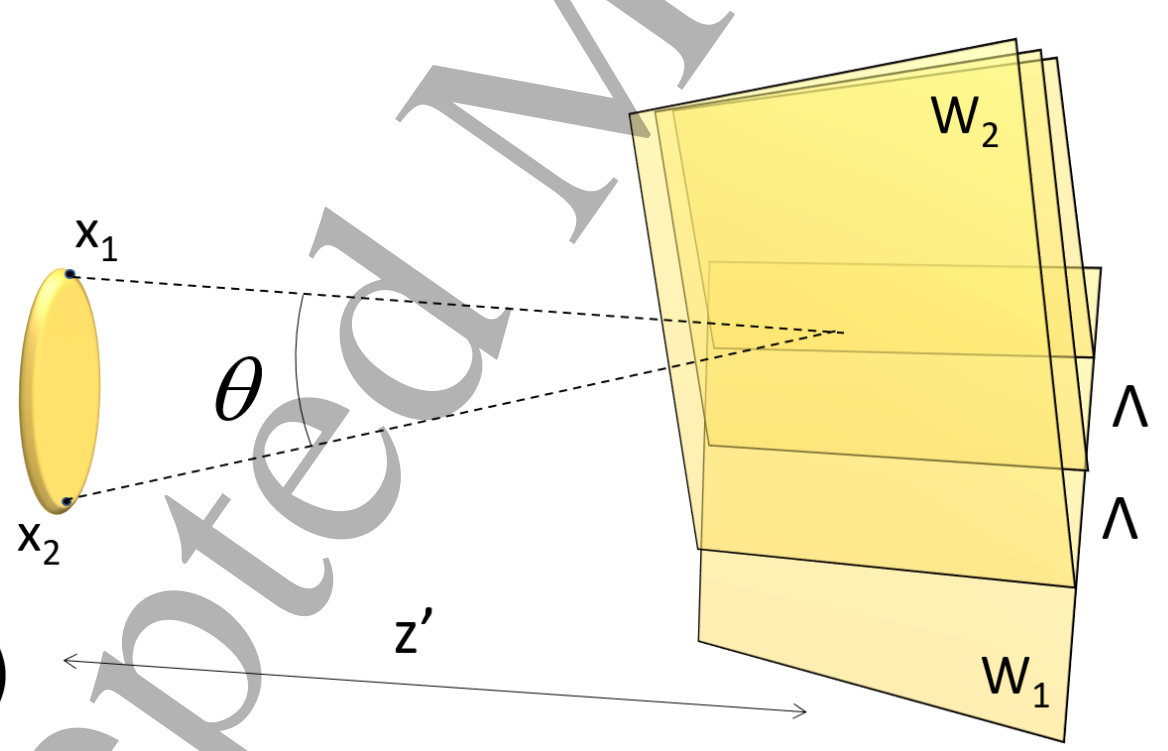

Figure 2. a) A source of linear extension D emits light impinging onto a plane at a distance $z$. Two pinholes diffract light towards an observation screen at a distance $z^{\prime}$ where the presence of interference fringes is analyzed. b) light emerging from the extrema $x_{1}$ and $x_{2}$ of the source fix the minimum spacing $\Lambda$ of the interference fringes formed by the two corresponding wavefronts $W_{1}$ and $W_{2}$ (here represented as plane waves). 
We can conclude that the illumination of the pinholes can occur in two opposite conditions [2,9]:

1) $d \ll \Lambda$ : fringes are present at the observation screen. The system is linear in the (complex) fields.

2) $d \gg \Lambda$ : the observation screen is uniformly illuminated. The system is linear in the intensity,

Eq. (9) allows to set up the illumination conditions to achieve a coherent illumination over a transverse size large enough. Let's exploit a common LED illuminator, from a mobile phone for example, about $2 \mathrm{~mm}$ in size and a wavelength $\lambda=0.5 \mu \mathrm{m}$ approximately. At a distance $L=$ $5 \mathrm{~m}$ the transverse coherence length is approximately $1.25 \mathrm{~mm}$. This can be considered as the minimum to perform the experiences described below. A larger distance would be better. Alternatively, by placing a diaphragm $1 \mathrm{~mm}$ in diameter at the source $L=5 \mathrm{~m}$ we will increase the coherence length as if we were further. In the following we will assume to have a coherence length of $2 \mathrm{~mm}$, specifically obtained in the examples reported below with a $2 \mathrm{~mm}$ LED at $L=$ $10 \mathrm{~m}$, if not differently specified.

\section{Fourier transforming with the naked eye}

The most common and available optical system is the human eye. It is composed of a lens (the crystalline) forming images onto a screen (the retina) that contains the sensible cells. In what follows the crystalline will be considered as a thin lens, the focal length being typically $f=17-18$ $\mathrm{mm}$. From this consideration it follows that transilluminating an object with a spatially coherent wave and looking through towards the source with the eye adapted to infinity, the retina will be illuminated by a light intensity distribution that is-the power spectral density, or power spectrum, of the wavefront transmitted by the object.

Quantitatively speaking, let the wave illuminating the object, $U_{0}$. It has uniform amplitude and phase over a plane of coordinates $(\xi, \eta)$, orthogonal to the propagation direction and placed at a distance $z<0$ from the lens. An object transilluminated by this wavefront introduces amplitude and phase modulations described by the complex function $T(\xi, \eta)$. The emerging field will then be:

$$
U(\xi, \eta)=U_{0} T(\xi, \eta)
$$

To measure the corresponding power spectrum we simply need to look through the object towards the source, with the eye adapted to infinity. The intensity distribution at the retina will then be given by:

$$
I(x, y)=|\mathfrak{F}[U(\xi, \eta)]|^{2}=\left|U\left(\lambda f f_{X}, \lambda f f_{Y}\right)\right|^{2}
$$

where $f$ is the crystalline focal length, $f_{\mathrm{X}}, f_{\mathrm{Y}}$ are the spatial frequencies of the plane waves the wavefront can be expanded into. By substituting the relations for the spatial frequencies in terms of the propagation angles of the plane waves with respect to the optical axis $z$, we have the linear positions on the retina:

$$
x=f \lambda f_{\mathrm{X}}=f \vartheta_{\mathrm{X}} \quad y=f \lambda f_{\mathrm{Y}}=f \vartheta_{\mathrm{Y}}
$$

(under paraxial approximation). This result is useful to make quantitative considerations from the observed power spectra. An easy "calibration" enables the observer to perform quantitative measurements in terms of spatial frequencies. By looking through the object with one eye, the other will be available to look at the source and its surroundings. Two advantages are achieved: a) both eyes will be infinity adapted, so that the Fourier transforming eye will operate properly; b) a scale placed in the source surroundings (better if orthogonal to the line of sight) will allow to measure 
the subtended angles $\vartheta_{\mathrm{X}}$ and $\vartheta_{\mathrm{Y}}$, and therefore the spatial frequencies $f_{\mathrm{X}}$ and $f_{\mathrm{Y}}$. We can perform quantitative measurements of Fraunhofer diffraction patterns with the naked eye.

It is useful to fix some numbers for the eye and the corresponding limits in viewing objects in terms of spatial frequencies. The angular resolution of the eye is commonly about $\vartheta_{\min } \sim 1 \operatorname{arcmin} \sim 310^{-4} \mathrm{rad}$. With a focal length $f=17 \mathrm{~mm}$ this corresponds to a transversal distance on the retina $x=f \vartheta_{\min } \sim 5 \mu \mathrm{m}$, or a spatial frequency $f_{0}=\vartheta_{\min } / \lambda \sim 6 \mathrm{~mm}^{-1}$ for a wavelength $\lambda=0.5 \mu \mathrm{m}$. A grating with such a spatial frequency will give diffraction maxima with the smallest distance perceived by the eye. The accessible range of spatial frequencies is reasonably extended to allow for experiences with common materials and objects to be Fourier transformed by the naked eye.

\section{Fraunhofer diffraction by apertures and obstructions}

Examples are provided here as suggestions to work with the Fourier transforming properties of the eye. In this section we will focus on the measure and characterization of the power spectrum. First, let's consider a small aperture, a pinhole with a diameter of a few $100 \mu \mathrm{m}$. It can be easily obtained by means of a sharp pin gently pressed onto a common thin Aluminum foil, better if placed onto a rigid plane. By looking through the hole illuminated by the LED source as detailed above, the corresponding Airy disk will appear with a number of satellite rings $[2,3,6]$. The angular position $\vartheta$ of the minima (zeroes) is related to the aperture diameter:

$$
D=1.22 \lambda / \vartheta
$$

that can be precisely measured according to the calibration described above. An example of a diffraction pattern from a calibrated circular aperture $300 \mu \mathrm{m}$ in diameter is shown in Fig. 3.a), here obtained through a digital camera instead of the eye. We stress that pictures are just reported as a rough example of the results, the vision with the naked eye giving much better insight into the actual diffraction patterns. We recall that the positions of the minima in the diffraction pattern of a circular aperture with diameter $D=2 a$ are given by the zeroes of the Bessel function, argument $x=k a \sin \vartheta$, occurring at $x=3.83,7.01,10.17,13.32,16.47$. Notice that they are not equally spaced.

By analyzing the rings and the corresponding minima positions compared to the reference scale, the first minimum occurs at a diameter of about $36 \mathrm{~mm}$. With $L=10 \mathrm{~m}$, this corresponds to $\vartheta_{1}=$ $1.810^{-3} \mathrm{rad}$, from which a diameter $D=340 \mu \mathrm{m}$ is obtained.

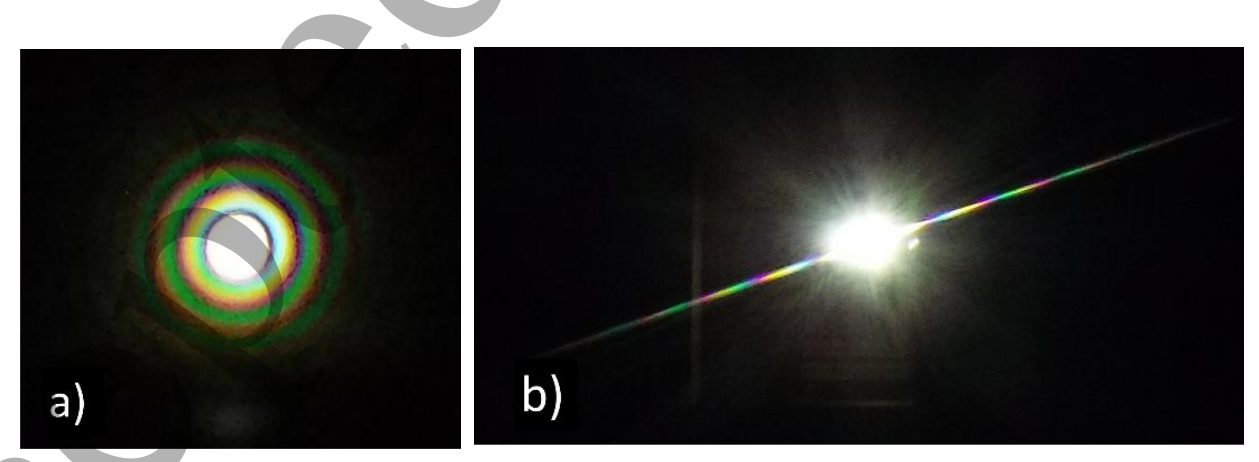

Figure 3. a) Fraunhofer diffraction pattern obtained with a calibrated circular aperture illuminated by white light from a LED $10 \mathrm{~m}$ far. b) the diffraction pattern of a hair. The angular scales are different in the two pictures, as explained in the text. 
A different example is represented by an obstruction, as easily obtained by studying the diffraction from a hair. In this case a linear diffraction pattern is obtained (see Fig. 3.b), extended in a direction perpendicular to the hair. Note that in this case the 1.22 prefactor in Eq. 13 is missing [2,3,10], since it comes from the Fourier transform of the circular aperture. For the first minima position at a distance $L=10 \mathrm{~m}$ we find values ranging from 50 to $90 \mathrm{~mm}$ from the center, depending on the hair diameter. Note that it is convenient to measure the distance of the null intensity. For example, a thin hair gives $85 \mathrm{~mm}$, corresponding to an angle $\vartheta_{1}=8.510^{-3} \mathrm{rad}$ and a diameter $D=60 \mu \mathrm{m}$ approximately.
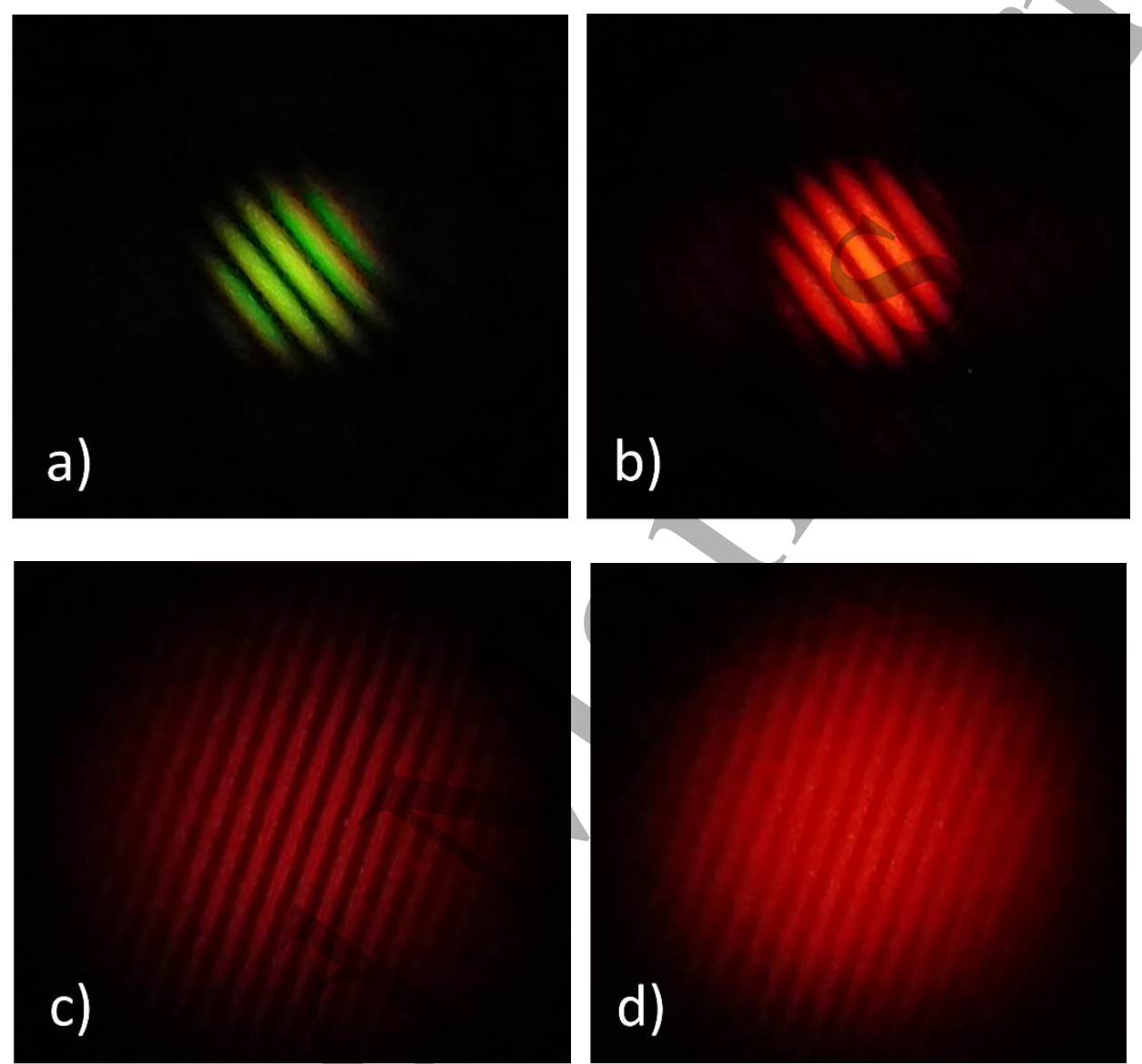

Figure 4. a) Young's experiment performed with two pinholes $0.3 \mathrm{~mm}$ spaced; b) the same as in a) with a red photographic filter: exposure time has been increased; c), d) the essence of the Wolf's experiment: two pinholes $0.8 \mathrm{~mm}$ spaced form interference fringes exhibit full spatial coherence at a distance $\mathrm{L}=8 \mathrm{~m}$ from the light source (c), and with a lower coherence length at a distance $\mathrm{L}=4 \mathrm{~m}(\mathrm{~d})$.

Looking through the aperture, the observer will notice the chromatic dispersion associated to diffraction of white light. This is well described by Eq. 13, where angle and wavelength are directly proportional. Therefore, a precise estimate of the minima positions could be an issue since minima occur at different angles in the diffraction pattern. Although slightly more demanding, a better result could be obtained by filtering the light from the white source with a proper bandpass filter. We notice that a narrow band is not so convenient, since it will dramatically reduce the light power making observation even more difficult. By contrast, a common photographic filter transmitting one color band will remarkably reduce enough chromatic dispersion effects. For a proper choice 
of the filter the emission wavelength spectrum of the white LED should be considered: the common peak around $450 \mathrm{~nm}$, given from the blue die of the device suggests a blue filter to be better than a green one, where the spectrum has a minimum. Also a red filter can be used, although a much wider spectral bandwidth is introduced by the yellow and red phosphors. These spectral features also explain why in the diffraction patterns of white LED light blue and red appear almost separated. Color dispersion, as well the advantages operating with a filter will apply to the experiences described below.

A special case of Fraunhofer diffraction is the Young's experiment. In [10] a clear discussion can be found. Originally exploited to give the proof of the wave nature of light, a very important extension has been introduced by Wolf, who exploited the same scheme to measure spatial coherence $[11,6]$. In Fig. 4.a) the result of a typical Young's experiment is shown with apertures constituted by two pinholes approximately $300 \mu \mathrm{m}$ in diameter, $1 \mathrm{~mm}$ spaced, obtained with a needle in a common thin Aluminum foil. In Fig. 4.b) a red filter is placed in front of the LED source. In Fig. 4.c) and 3.d) a similar experience is repeated at two distances, $L=8 \mathrm{~m}$ (c) and $L=$ $4 \mathrm{~m}$ (d) from the light source respectively, thus making the transverse coherence length changing from larger to slightly smaller than the pinhole spacing. The ultimate meaning of coherence is evidenced here, the fringes loosing their visibility when coherence is reduced (d). By systematically measuring the visibility of fringes as a function of the pinhole spacing, a measurement of the degree of coherence at a given distance from the source is possible. The widespread realm of coherence stems from this apparently simple observation, as it is clear by referring to the works by Wolf himself [11], or to the introduction of the Michelson stellar interferometer [7].

\section{Thin sinusoidal amplitude grating}

Although it might be considered a bit strange, a grating imposing a sinusoidal amplitude profile onto the transilluminating wavefront actually represents the ultimate grating: any transmission function can be thought to be the sum of a proper number of these gratings accordingly to the Fourier theorem. That's why we discuss this ideal transmission function. Despite its apparent peculiarity, it can be obtained quite easily with some trials on several proper samples. A grating imposing sinusoidal amplitude modulations across the plane wavefront generates a $0^{\text {th }}$ order maximum and the first order maxima only [2]. Thus, it is quite easy to recognize it just from its power spectrum, that is the Fraunhofer diffraction pattern. For example, such a grating can be likely well approximated by a simple tissue, with warp and weft acting as two perpendicular gratings. Different tissues can be tested, by looking for the cases where the number of diffraction maxima is minimum, ideally one. In such a case we have evidence that the system behaves as we need. The material depth changes across each thread, thus introducing an oscillating smooth wavefront modulation mimicking a sinusoidal grating. Fig. 5.a) shows the Fraunhofer diffraction pattern from a common thin white towel. The Fraunhofer diffraction from an (almost) ideal sinusoidal grating is shown in Fig. 5.b), as obtained with the same setup above. Realizing good sinusoidal gratings is not so difficult by taking pictures with an old-fashioned film camera of a proper printed pattern. By means of computer-generated pictures, a convenient greytone profile can be obtained on the film to impose sinusoidal modulations onto a plane wave. Taking pictures in conditions of bright illumination and perpendicularly to the mask will produce proper gratings on the film with a groove distance that can be simply imposed by changing the camera-picture distance. Care has to be taken to take pictures with proper exposure times. In [2] the realization of computer generated holograms with photopolymers is extensively described.

Notice that a comparison can be done with the most common Ronchi ruling, or Ronchi mask. This is a peculiar widely spaced grating (order of magnitude 100 microns) characterized by apertures and stops of the same size, with sharp edges. A detailed discussion about the Ronchi ruling and its applications in Optics can be found in [12] (in Italian). Illuminated by a plane wave it generates a

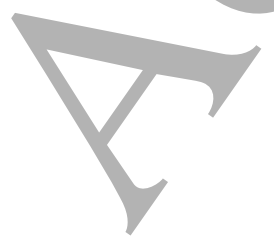


3

4

5

6

7

8

9

number of diffraction orders due to the large spacing and because of the sharp borders that introduce Fourier components even at high spatial frequencies. In Fig. 5.c) the Fraunhofer diffraction pattern of a Ronchi ruling is reported. Here the chromatic dispersion, increasing with the order number, is much more evident than in the previous case. Finally, in Fig. 5.d) the same measurement is shown by red filtering, as already done in Fig. 4.

For the sake of experimental simplicity, we will consider hereinafter the tissue producing one diffraction order as the benchmark for gratings in almost all the experiments described in the following sections.
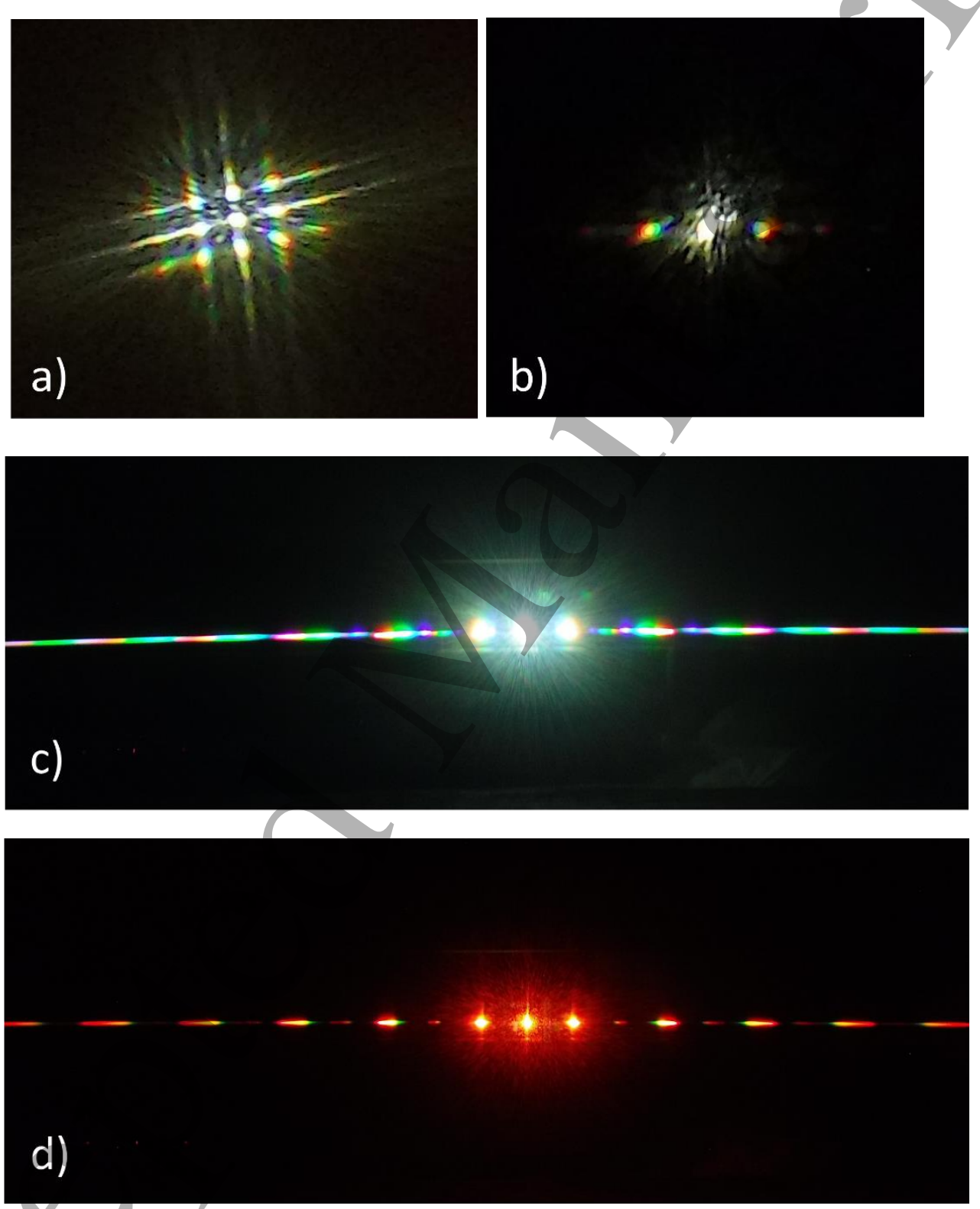

Figure 5. Fraunhofer diffraction patterns from three examples of grating. a) common thin towel, acting as a 2D grating; b) an (almost) ideal sinusoidal grating obtained as described in the text; c) a laboratory Ronchi ruling; d) the same Ronchi ruling, illuminated with filtered red light. All measurements have been obtained with the same optical setup described above. 


\section{Basic Fourier transform theorems}

The naked eye exploited as a device for Fourier transforming of transmission functions allow to realize simple experiences reproducing the basic Fourier analysis theorems. Since we always access the power spectrum of the transmission function, some issues have to be considered when interpreting diffraction patterns, as we point out below.

\subsection{Similarity theorem}

Let it be $G\left(f_{\mathrm{X}}, f_{\mathrm{Y}}\right)=\mathfrak{F}[g(x, y)]$; then:

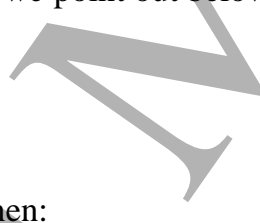

$$
\mathfrak{F}[g(a x, b y)]=\frac{1}{|a b|} G\left(\frac{f_{\mathrm{X}}}{a}, \frac{f_{\mathrm{Y}}}{b}\right)
$$

Let's focus at the diffraction from the tissue, fixing the positions of the diffraction maxima. If we now slightly stretch the tissue in one direction it will be elongated but will also shrink in the perpendicular one. The diffraction pattern will show the opposite behavior: maxima will be contracted in the direction of the tissue elongation, while the distance increases in the perpendicular one. In terms of the theorem of above, stretching the coordinates $(x, y)$ in the direct space will cause a contraction of the coordinates in the frequency domain. A change in the amplitude of the Fourier components is also present (almost invisible in our experience).

Generally speaking, the similarity theorem ultimately explains the complementary role of Fourier analysis in either the direct or the Fourier space. In brief, phenomena that are too small, or fast, to be studied in the direct space will easily be characterized in the Fourier domain, and viceversa. This is a simple example of the relations among conjugate variables in waves, that has its counterpart in the Heisemberg's uncertainty principle in Quantum Mechanics $[1,4]$. 


\subsection{Linearity theorem}

Let it be $G\left(f_{\mathrm{X}}, f_{\mathrm{Y}}\right)=\mathfrak{F}[g(x, y)]$ and $H\left(f_{\mathrm{X}}, f_{\mathrm{Y}}\right)=\mathfrak{F}[h(x, y)]$; then:

$$
\mathfrak{F}[a g(x, y)+b h(x, y)]=a G\left(f_{\mathrm{X}}, f_{\mathrm{Y}}\right)+b H\left(f_{\mathrm{X}}, f_{\mathrm{Y}}\right)
$$

Despite its essential simplicity, the realization of this theorem requires some attention. We need a transmission function that is the sum of two given functions. It is common for the students to consider two hairs or even the tissue itself as good candidates for this experience, due to the apparently clear superposition of two or more obstructions. It must be noticed that this superposition is not actually a sum, but a product. By contrast, two separate apertures (e.g. slits) will be suitable to the aim. Notice that if they superimpose, again the resulting function is a product. Unfortunately, producing two or more separate apertures small and close enough to realize the experience is quite difficult (except for the case of pinholes in the Young's experiment discussed above). To overcome this difficulty, we invoke the Babinet's Principle, stating that the fields diffracted by an aperture and the complementary obstacle have identical amplitudes, opposite phases. Therefore, we can safely operate with two separate obstructions, thus generating the same fields that would have been generated by two apertures. The two fields sum and form the corresponding power spectrum. We illuminate two hairs, preferably of different diameter, better if not crossing neither parallel, close enough to fall within one coherence area. In Fig. 6.a) we report the result of such a measure. Hairs can be easily measured independently, and the corresponding diffraction patterns easily recognized thus evidencing the linearity theorem.

Two comments are worth here. We actually realize the sum of two fields with the "wrong" object, actually representing the product of two functions. Since we are interested in summing the fields, we can still operate correctly thanks to the Babinet's principle. Clearly the interpretation is crucial here, and the product case must be considered properly, as will be discussed with the next experience. The second point is that we access the power spectrum, that is the squared modulus of the sum of the two fields. Therefore, cross terms arise that give origin to additional, much fainter maxima (invisible in Fig. 7.a)). The case of parallel hairs, or slits, better evidence these terms, being a revisited version of the Young's experiment. Again, provided that proper description is used to interpret data, the non-trivial effect of the interference pattern becomes an evident result of the linearity theorem.

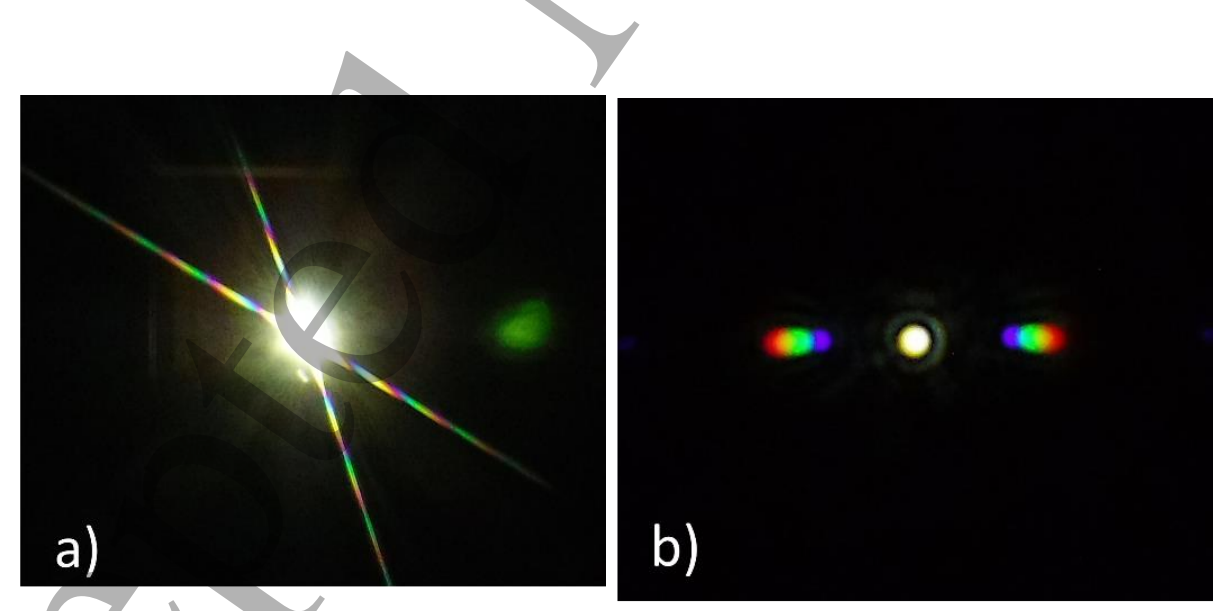

Figure 6. a) Linearity theorem: example of the diffraction pattern generated by two hairs forming an angle; b) convolution theorem: the diffraction pattern generated by the Ronchi ruling through a diaphragm (scale is different from Fig. 4.c). 


\subsection{Convolution theorem - resolution}

Let it be $G\left(f_{\mathrm{X}}, f_{\mathrm{Y}}\right)=\mathfrak{F}[g(x, y)]$ and $H\left(f_{\mathrm{X}}, f_{\mathrm{Y}}\right)=\mathfrak{F}[h(x, y)]$. Then:

$$
G\left(f_{\mathrm{X}}, f_{\mathrm{Y}}\right) H\left(f_{\mathrm{X}}, f_{\mathrm{Y}}\right)=g(x, y) * h(x, y)=\iint g(\xi, \eta) h(\xi-x, \eta-y) \mathrm{d} \xi \mathrm{d} \eta
$$

that is the convolution of the two functions. This theorem has several applications, as we will briefly discuss below. A number of combinations between transmission functions will give a clear realization of this theorem. We just introduce a couple of cases that could be interesting also in view of more general applications.

The simplest realization of this theorem is given by something we already discussed above, the diffraction from a circular pinhole, for example. In its extreme simplicity, it shows that the incoming plane wave, multiplied by the aperture function will generate a Fraunhofer diffraction pattern given by the squared modulus of the convolution between the two Fourier transforms: the Dirac delta and the Bessel function, respectively.

The previous example might appear too simplicistic, as the convolution by a Dirac delta is ultimately a product. A slightly more interesting realization can be obtained by superimposing a grating and an aperture. For example, an aperture used as a diaphragm in front of the tissue, or the Ronchi ruling. The aperture must be properly sized to let at least some threads, or grooves, to be illuminated and diffract the incoming light. In this case each narrow spot formed by the grating will be enlarged to a size given by the power spectrum of the aperture itself. Again, both power spectra can be analyzed separately and compared to the power spectrum of their combination. Fig. 6.b) reports the result obtained with a Ronchi ruling and the small diaphragm adopted for obtaining the pictures shown in Fig. 3.

As mentioned above, the transmission function imposed by the tissue is the product of two independent functions imposed by the two perpendicular gratings represented by warp and weft. The corresponding Fraunhofer diffraction pattern will then be given by squared modulus of the convolution between the two Fourier transforms. One can easily prove that this will give a diffraction pattern composed by the pattern of one grating, for example the warp, extended in one direction, repeated in the perpendicular direction as many times as the Fourier transform of the weft will have its diffraction orders: the convolution is then realized. This is identical to the case of two gratings summed up (for example, by displacing them in separate positions of the plane), thus explaining the ambiguity mentioned above.

Besides the basic interpretation in terms of convolution, the results obtained so far also introduce to a fundamental property of any optical system: the concept of resolution. Without entering into details about this item, we will simply note that by introducing a diaphragm the diffraction pattern is affected by diffraction of the aperture itself. This limits the capability of the system to distinguish or resolve close maxima. Because the Fraunhofer diffraction pattern can be considered as one of the basic schemes for imaging, we understand how optical systems are limited in resolution by their geometrical apertures. The Rayleigh criterion, for example, states that the ultimate limit for distinguishing two pointlike sources is ideally obtained with the first diffraction minimum of the image of the former corresponding to the central maximum of the latter. It is an important practical estimate, but a more detailed analysis is needed as the noise occurring in the system constitutes the ultimate limitation for the resolving power. Finally, It is important noticing that it is not straightforward to extend the result obtained here above to the loss in resolution imposed by looking with the naked eye through a diaphragm: in this case the wavefront falling through is not spatially coherent, and the image formation is not given by the squared modulus of the convolution, as above. We let the reader referring to textbooks for a description of this difference. 


\subsection{Shift theorem}

If $G\left(f_{\mathrm{X}}, f_{\mathrm{Y}}\right)=\mathfrak{F}[g(x, y)]$, then:

$$
\mathfrak{F}[g(x-a, y-b)]=G\left(f_{\mathrm{X}}, f_{\mathrm{Y}}\right) \exp \left[-i 2 \pi\left(f_{\mathrm{X}} a+f_{\mathrm{Y}} b\right)\right]
$$

That is, a linear translation is described by a phase shift in the Fourier space, the phase shift of a given Fourier component being proportional to the spatial frequency. This is almost obvious by thinking to the phase shift to be introduced into the argument of an oscillating function to translate it by a given distance.

The realization of this theorem can be obtained in two ways. The former is by simply translating any aperture or object in front of the naked eye and looking to its power spectrum. As already noticed above, it will be unchanged. Now, by noticing that the transmission function changes upon translation, one immediately concludes that only phases can change, since the amplitudes are maintained. Nevertheless, this can be unsatisfactory, or incomplete, since the phase changes cannot be measured.

The latter realization needs some care and is perhaps the most difficult experience described here. It can still be done with simple components as above, but requires a very careful preparation and usually a number of trials and errors. Ultimately, since we are going to measure phases, an interferometric measurement is necessary. In order to prevent the students from a very difficult procedure, it is much better to operate with two identical Ronchi rulings or at least two custommade sinusoidal gratings having 20-30 lines/mm.

Let's place the two identical gratings along the optical path, separated by a distance of approximately $5-10 \mathrm{~mm}$. Both generate a $0^{\text {th }}$ order and two symmetrically $1^{\text {st }}$ order diffracted waves, the latter grating being illuminated by the plane wave transmitted by the former. Provided that the gratings are identical, the diffracted beams superimpose along the same directions. They are two identical plane waves with similar amplitude, so they will interfere depending on the relative phase. This phase difference is imposed by i) the grating distance ii) the relative position of the grating in the transversal plane, according to the shift theorem itself.

Therefore, a condition will exist when the wave diffracted by the former will have opposite phase with respect to the latter, and they will cancel out. It is straightforward to find that the same condition will give the opposite waves with the same phase, and constructive interference will be there. As a result, one will observe an asymmetric Fraunhofer diffraction pattern with one maximum instead of two symmetric maxima.
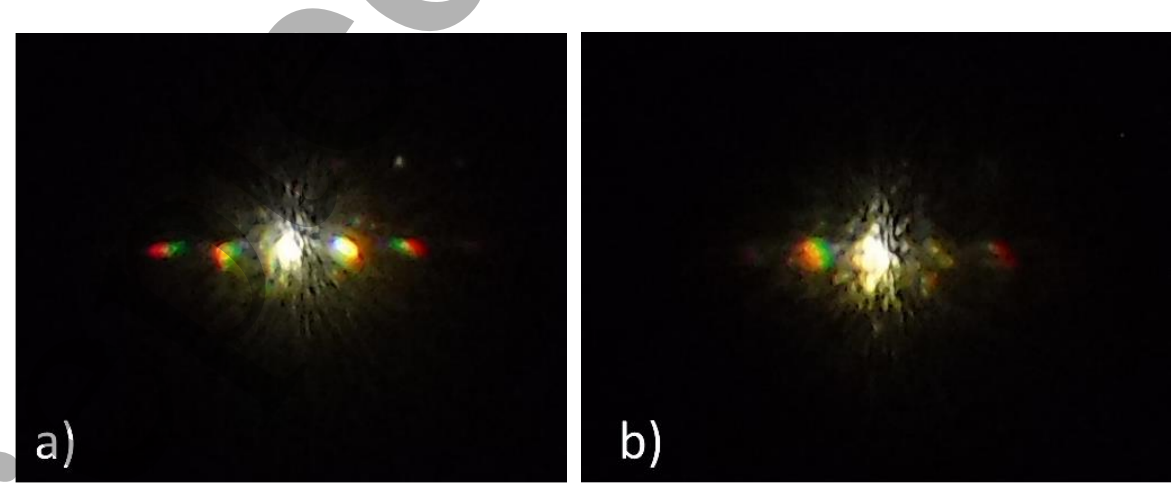

Figure 7. a) Diffraction pattern obtained with two identical sinusoidal amplitude gratings 5 $\mathrm{mm}$ spaced along the optical axis. In b) the phase difference between the two gratings is such that diffracted beams on one side (right in the picture) have opposite phases and cancel out by destructive interference. 
To reach this condition the experimenter will have to move very carefully one grating transversally to the optical axis. By doing this, maxima will disappear alternatively on opposite sides. If a micrometric stage is adopted to translate the grating, the measurement of the transversal distance between two positions where one maximum disappears will provide the grating spacing itself. This is in quantitatively accordance with the shift theorem. Fig. 7 shows an example of this condition obtained with two identical sinusoidal gratings. In a) both maxima are present; in b) the condition is reached when one maximum is there, the opposite being cancelled by destructive interference due to the relative phase.

The effect evidenced by this experience goes well beyond the simple realization of the shift theorem. We have obtained an asymmetric diffraction pattern, in open contrast with any other diffraction pattern encountered so far. This is related to another fundamental property of the Fourier transform: if either the imaginary or the real part of the input functions is zero, the Fourier transform is strictly symmetric. Any 2D amplitude modulation as those considered here can be considered as a real-valued function provided that it is properly illuminated by a plane wave. On the contrary, in the case of two gratings displaced along the optical axis, this is not always true and the Fourier transform might be asymmetric. This is the essence of the additional condition bringing to Bragg diffraction for three dimensional gratings $[2,3]$.

\section{The $4 \mathrm{f}$ system and the Fourier integral theorem}

A remarkable step forward is possible by introducing an optical scheme of huge importance and utility in many applications of coherent Optics. This is represented by the so called $4 f$ system, schematically depicted in Fig. 8, slightly adapted to our case: the former lens with focal length $f_{1}$ operates a first Fourier transform of the wavefront; the latter, focal length $f$ (here the eye), collects the Fourier spectrum and operates another Fourier transform. Ideally, the $4 f$ system has two equal lenses spaced by twice the focal length: it collects the wavefront from the front focal plane of the first lens and double transforms it at the back focal plane of the latter. Hence the name. Following Eq. (7), at the back focal plane of the latter lens we then obtain a wavefront that is given by:

$$
U(u, v)=\mathfrak{F} \mathfrak{F}[U(\xi, \eta)]
$$

where $U(\xi, \eta)$ is the wavefront at the entrance focal plane of the system, $U(u, v)$ at the exit focal plane.

By considering this result in terms of fields, we notice that the double transformation has an immediate interpretation. $\operatorname{Let} G\left(f_{X}, f_{y}\right)=\mathfrak{F}[g(x, y)]$, then it follows:

$$
\mathfrak{F}\left[G\left(f_{\mathrm{X}}, f_{\mathrm{Y}}\right)\right]=\iint G\left(f_{\mathrm{X}}, f_{\mathrm{Y}}\right) \exp \left[-i 2 \pi\left(x f_{\mathrm{X}}+y f_{\mathrm{Y}}\right)\right] \mathrm{d} f_{\mathrm{X}} \mathrm{d} f_{\mathrm{Y}}=g(-x,-y)
$$

Thus, the double transformation of the field gives the field itself with inverted coordinates. If, instead of Fourier transforming the spectrum $G\left(f_{\mathrm{X}}, f_{\mathrm{Y}}\right)$ it would be inverse transformed, the function $g(x, y)$ will be obtained according to the integral Fourier theorem. This also draws the attention to the strict similarity existing between Fourier transform and inverse transform, that are ultimately the same operator.

A comment is worth here about the case when the two focal lenses are different, as it will likely be in our system. Then the similarity theorem plays its role, by bringing to the results that a simple scaling is there between the spatial coordinates in the two fields. Precisely, coordinates are scaled as the ratio of the focal lengths.

As a consequence, we recognize the $4 f$ system, or its evolution with different focal lengths, as a realization of this fundamental theorem of Fourier analysis. In our experience, the latter lens is 
represented by the eye crystalline, with the retina placed in the corresponding back focal plane $(u, v)$.

We need to introduce an additional lens to perform the first Fourier transform of the field. To this aim we exploit a simple magnifying lens, also named as a lupe. Any positive lens will work, of course, provided that the focal length is not much larger than the eye. The mounting typically present in these objects helps the experimenter, as it will be clear below.

First, we need to fix the front and back focal planes. By simply imaging an object far enough (ideally at infinity) and well illuminated onto a thin white paper we immediately find both. In Fig. 9.a) the magnifying lens is shown to produce images at both focal planes. Let's then build two tubes, preferably black inside to avoid or almost reduce spurious reflections (stray light). The lengths of these tubes should be such that they finish exactly at the front and back focal planes. We can finally check this by imaging objects at infinity once more. In Fig. 9.b) we show an example of the mounted system.

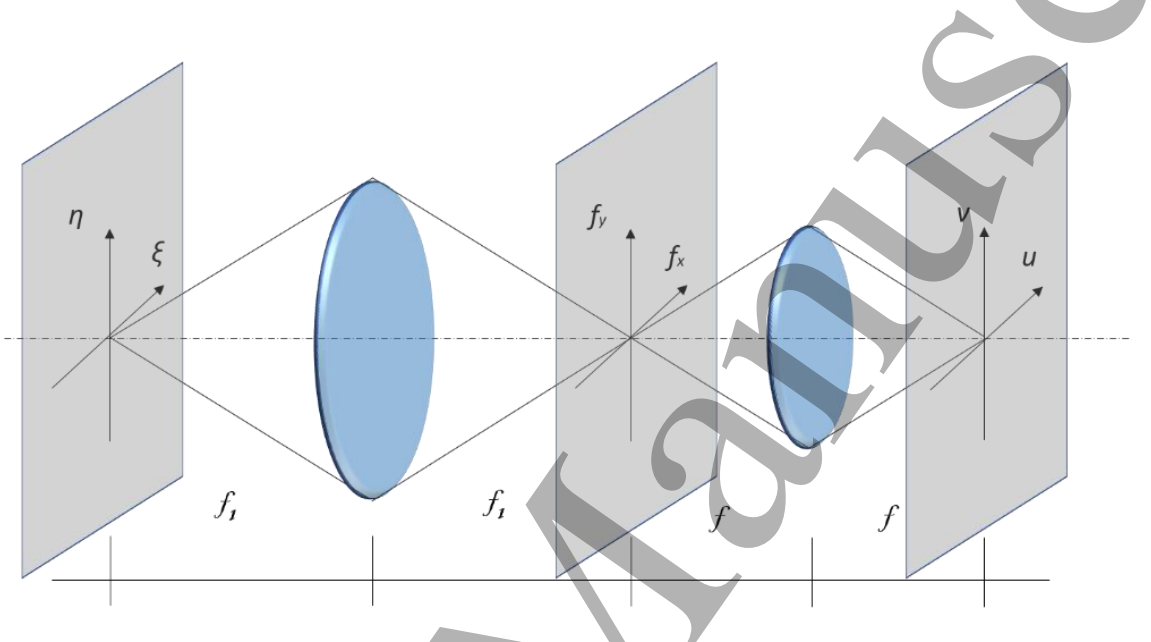

Figure 8. Schematic of the modified $4 f$ system described in the text. Two Fourier transforms are operated in sequence.

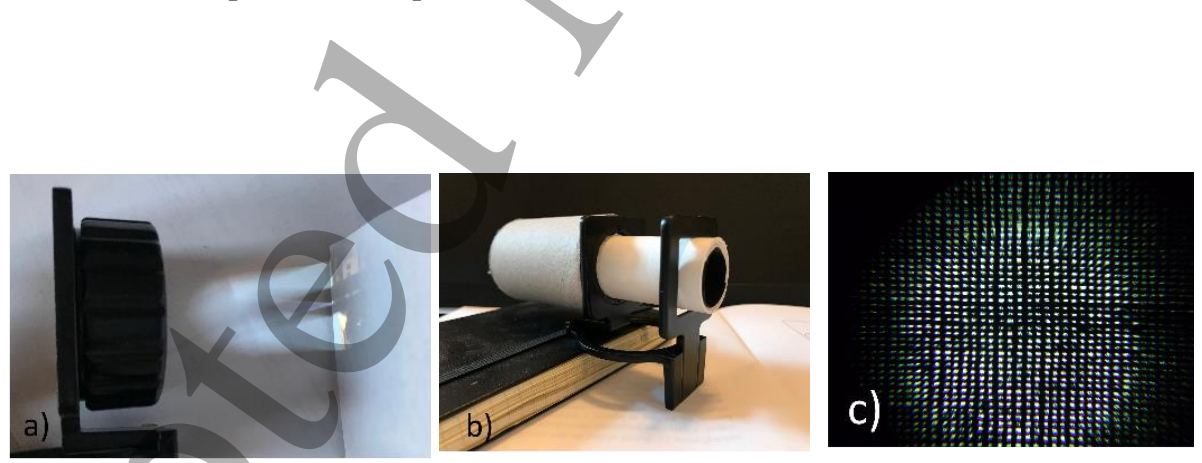

Figure 9. a) the magnifying lens forming infinity conjugated images; b) the final system mounted; c) an example of the reconstruction of the field through the $4 \mathrm{f}$ optical system.

Now we are ready to realize the $4 f$ configuration. With the light source at the convenient distance, as above, we put an object in the front focal plane and look into the system with the eye just downstream the back focal plane: it will operate the latter Fourier transform. By adapting the eye at infinity, we will see the intensity distribution as transmitted by the object. Fig. 9.c) shows an 
example of this result for the tissue of above. A replica of the object will be seen, accordingly to the Integral Fourier theorem.

Further experiences can be performed by using this device with many objects. Accurate observation will prove that the reconstructed field, and the corresponding intensity distribution, meet the scaling condition mentioned above, accordingly to the ratio of the focal lengths.

\section{Spatial filtering}

The $4 f$ system introduces opportunities to operate on wavefronts. Accessing the Fourier spectrum in the intermediate focal plane $\left(f_{\mathrm{X}}, f_{\mathrm{Y}}\right)$ allows to introduce the so-called spatial filtering, thus opening the way to a widespread variety of experiences. Here we just focus on a couple of applications that are worth to be discussed in a basic Fourier Optics course. Many other applications can be found and realized, as briefly discussed below.

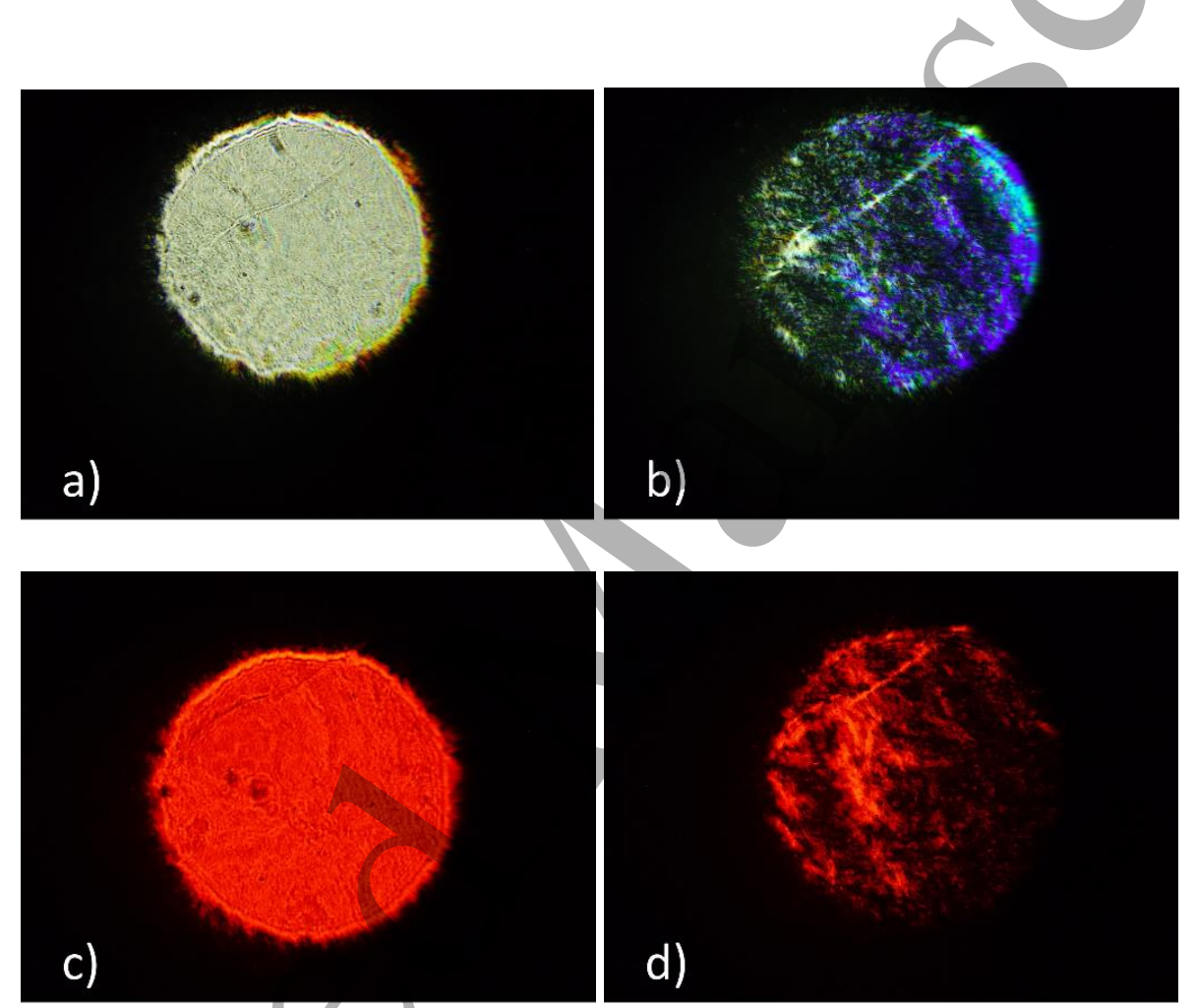

Figure 10. a),b) example of single side band filtering of a wavefront emerging from a thin plastic film: the phase modulations introduced by transilluminating a thin plastic film give negligible intensity modulations in a) while they become evident in b). In c), d) the same is repeated with filtered red light.

In principle, operating on the Fourier spectrum of a wavefront can be done in many ways, by introducing amplitude modulations, phase modulations, or both in the intermediate focal plane. For the sake of experimental accessibility, here we focus at amplitude modulations only, that are relatively easy to be implemented with our system. Therefore, we can introduce high-pass, low pass, bandpass, single-side band filters in the 2D spectrum. Low pass filtering can be easily obtained by introducing a small diaphragm in the intermediate focal plane. High pass filtering can be easily obtained by introducing a thin wire: notice that this also removes the transmitted beam, 
thus realizing a configuration similar to that adopted in the dark field imaging, often used in microscopy. We refer to specific texts to describe this application. We report here an example of the single side band filtering, adopted to visualize phase objects as it is for example in Schlieren photography. In Fig. 10.a) we report the $4 f$ intensity distribution obtained by transilluminating a thin plastic film, to be compared to $b$ ) where a knife edge has been introduced vertically in the intermediate focal plane to remove half of the Fourier spectrum. Phase modulations invisible in a) become evident. In c) and d) the same experience is repeated by inserting the photographic red filter. Notice that characterizing the intensity distributions obtained in such experiences does need a digital camera properly calibrated. An example of camera calibration is given in [13].

\section{The Abbe-Porter Experiment (revisited)}

One of the most insightful applications of spatial filtering and Fourier Optics in general is the Abbe-Porter experiment, which elucidates very clearly the Abbe theory for the interpretation of coherent image formation. Actually, we cannot realize this experiment in its original form, but a revisitation based on pure spatial filtering is easy to be performed with our $4 f$ system. Basically, Abbe's theory describes the image formation through a lens as a specific interpretation of the fields in its back focal plane. Considering the wavefront entering the lens as its Fourier decomposition in plane waves immediately shows that the back focal plane is characterized by pointlike sources, one for each plane wave accordingly to the Fourier transform. Plane waves can be interpreted as due to the beam diffracted by sinusoidal amplitude gratings at the object plane (see above). A plane wave propagating along the optical axis is formed as the superposition of the corresponding $0^{\text {th }}$ order diffracted waves from all the gratings: it focuses on the optical axis. On the image plane spherical waves emerging from these sources interfere with the transmitted, forming sinusoidal fringes: the image is then formed by the superposition of simple interference patterns, as the object has been assumed to be decomposed in terms of sinusoidal gratings. In our $4 f$ configuration the scheme is basically different: the field diffracted by the object is transformed twice. Therefore, we cannot realize the original Abbe-Porter experiment. Nevertheless, the spatial filter in the intermediate focal plane evidences the Fourier decomposition in plane waves evident and allows to operate in the Fourier space as it is done in the original Abbe-Porter experiment.

For example, realizing the Abbe-Porter experiments can be done as described here below. A 2D grating is imaged and a thin slit placed in the back focal plane of the lens parallel to one direction of the grating grooves operates a 1D low-pass filtering. Only the central portion of the 2D spectrum is let to propagate further, producing the interference with the transmitted field. Interference fringes then degenerate along the direction perpendicular to the slit. We can introduce a similar filtering by placing the slit in the intermediate focal plane of the $4 f$ system, that is in front of our eye. The image of a 2D grating formed on the retina will then be composed by continuous lines perpendicular to the slit direction. The image is clearly missing the Fourier components needed to form the image of the grating in the other direction. In Fig. 11 the result of this experience is shown: in a) the $2 \mathrm{D}$ grating is reproduced through the $4 f$ system, in b) the wavefront from the same grating is passed through a slit placed in the intermediate focal plane. Again, in c) and d) we operate with red light. In e) and f) the same experience is repeated with the $2 \mathrm{D}$ grating rotated around the propagation direction: still, horizontal lines are only present according to the slit position, irrespectively of the grating position.

The Abbe-Porter experiment is only one among a number of applications of spatial filtering that constitute the realm of Optical Information Processing: examples are the Zernike's phase contrast method, Schlieren imaging (just mentioned above), Marechal's photography applications, character recognition through the Vander Lugt filter, etc. Developed overall the twentieth century, these methods have been reconsidered when developing the computer-based image processing, widely used in a widespread class of software and applications to work on digital pictures. 

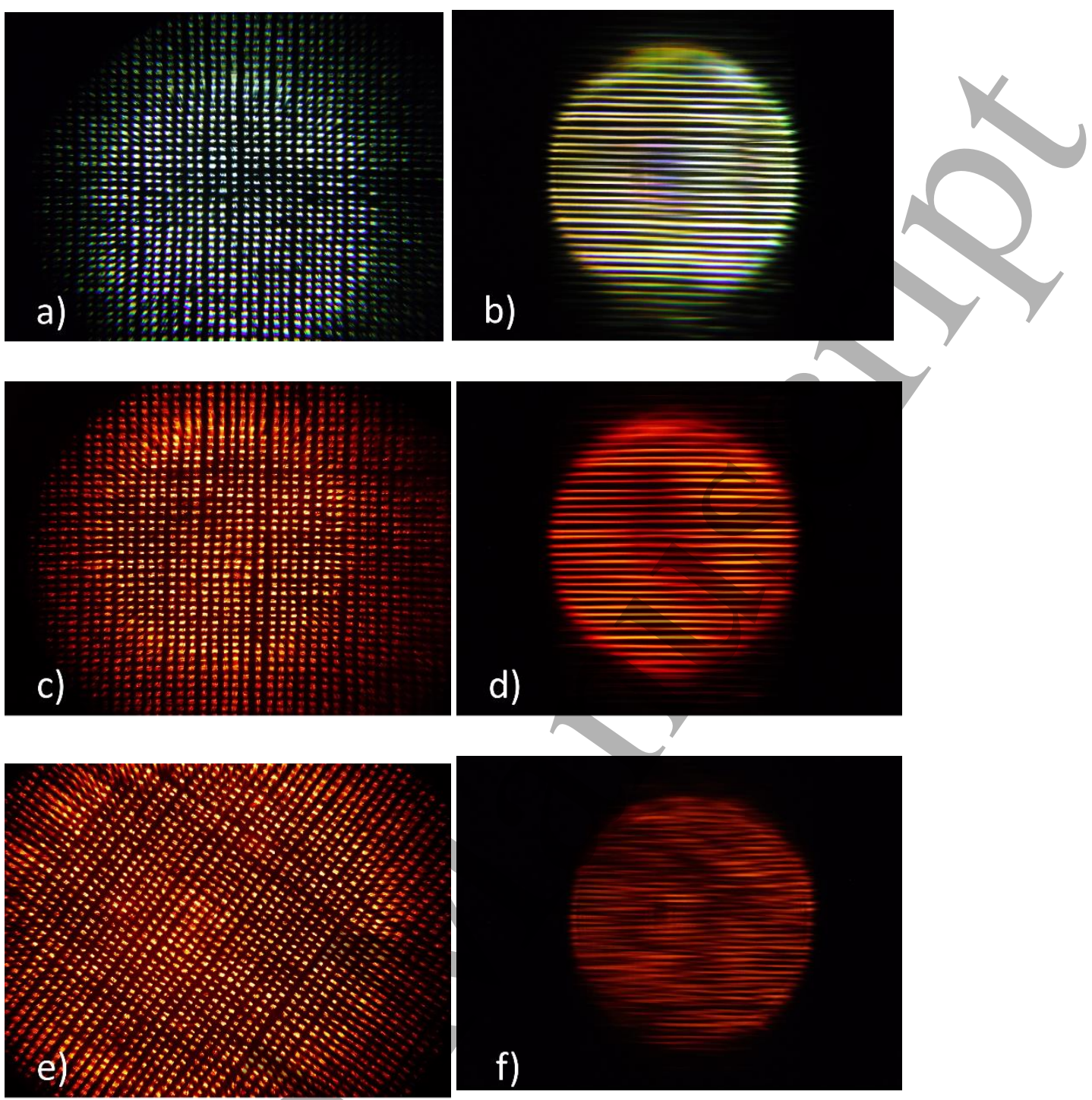

Figure 11. Revisitation of the Abbe-Porter experiment. In a) the wavefront from a $2 \mathrm{D}$ grating is passed through the $4 \mathrm{f}$ system; in b) it is filtered by blocking all diffraction orders along the horizontal direction. In c), d) the same is repeated with the red filter. In e) the 2D grating is rotated and in $\mathrm{f}$ ) the same horizontal filtering is operated, thus forming horizontal lines that are not in the object.

\section{Conclusions}

Approaching Fourier analysis through simple experiences is of huge importance for undergraduate students, who are going to exploit these methods in a widespread range of applications, both within theory, experiments and numerical analysis. An example of numerical computation can be found in [14], where a number of numerical experiments can be easily performed. Here I have introduced some very simple experiences to be proposed to a class of students. Experiences can be performed by single students who will practice in preparing an experiment and observing the relevant 
elements of a relatively complex resulting effect. Despite the extreme simplicity of the "apparatus", accurate preparation allows to enter into specific applications of the theorems and applications. Moreover, the experiences described in this work can be easily done without accessing any lab, even at home with common materials and objects. Improving the same experiences in an equipped lab might be a very interesting approach for further practicing with laboratory equipment, but it is not essential. Last but not least, the experiments suggested here introduce no hazards at all, even if exploiting the naked eye, making it really safe to let students operating alone. This makes them appropriate also for high school students, although the formal approach might be properly reduced.

Using a LED light allows to enter into the details of coherence, a matter of remarkable importance in physics. This is interestingly related to Fourier analysis, too, exhibiting the complementary realms of spatial and temporal coherence. These arguments can also be adapted to lab experiences for graduate students.

\section{Acknowledgments}

I thank Francesco Ragusa for suggesting to build a laboratory to teach the fundamentals of Fourier analysis more than ten years ago, and Marzio Giglio for almost two decades of discussions about Fourier optics. I thank the two anonymous referees for useful suggestions.

\section{References}

[1] Manrusipur M 2020 Appl. Optics 591559

[2] Goodman J W 2017 Introduction to Fourier Optics, 4th ed. (W.H. Freeman and Company)

[3] Rossi B B 1957 Optics (Addison-Wesley Publishing Company)

[4] Feynman R P, Leighton R B, Sands M 1964 The Feynman Lectures on Physics Vol. II (Addison-Wesley)

[5] Goodman J W 2000 Statistical Optics (John Wiley \& Sons Inc.)

[6] Born M and Wolf E 2002 Principles of Optics, 7th ed. (Cambridge University)

[7] Michelson A A and Pease F G 1921 Astrophys. J. 53249

[8] Illaramend M A et al 2014 Am. J Phys. 82649

[9] Zernike F 1938 Physics (Amsterdam) G 78

[10] Monk G S 1963 Ligth: Principles and Experiments (New York : Dover Publications Inc.)

[11] Thompson B J and Wolf E 1957 JOSA 47895

[12] Toraldo di Francia G 1958 La diffrazione della luce (Torino: Einaudi)

[13] Potenza M A C 2015 Eur. J. Phys 36065040

[14] https://www.epfl.ch/schools/sb/research/iphys/teaching/crystallography/diffraction-fourier-transform/ 